\title{
A non-iterative approach to frequency estimation of a complex exponential in noise by interpolation of fourier coefficients
}

DOI:

10.1109/ICDSP.2013.6622758

Link to publication record in Manchester Research Explorer

Citation for published version (APA):

Minhas, S. F., \& Gaydecki, P. (2013). A non-iterative approach to frequency estimation of a complex exponential in noise by interpolation of fourier coefficients. In 2013 18th International Conference on Digital Signal Processing, DSP 2013/Int. Conf. Digit. Signal Process., DSP https://doi.org/10.1109/ICDSP.2013.6622758

Published in:

2013 18th International Conference on Digital Signal Processing, DSP 2013|Int. Conf. Digit. Signal Process., DSP

\section{Citing this paper}

Please note that where the full-text provided on Manchester Research Explorer is the Author Accepted Manuscript or Proof version this may differ from the final Published version. If citing, it is advised that you check and use the publisher's definitive version.

\section{General rights}

Copyright and moral rights for the publications made accessible in the Research Explorer are retained by the authors and/or other copyright owners and it is a condition of accessing publications that users recognise and abide by the legal requirements associated with these rights.

\section{Takedown policy}

If you believe that this document breaches copyright please refer to the University of Manchester's Takedown Procedures [http://man.ac.uk/04Y6Bo] or contact uml.scholarlycommunications@manchester.ac.uk providing relevant details, so we can investigate your claim.

\section{OPEN ACCESS}




\section{A Non-Iterative Approach to Frequency Estimation of a Complex Exponential in Noise by Interpolation of Fourier Coefficients}

\author{
Shahab Faiz Minhas* \\ School of Electrical and Electronics Engineering \\ University of Manchester \\ Manchester, UK \\ e-mail: shahab.faiz@manchester.ac.uk
}

\author{
Patrick Gaydecki \\ School of Electrical and Electronics Engineering \\ University of Manchester \\ Manchester, UK \\ e-mail: Patrick.gaydecki@manchester.ac.uk
}

\begin{abstract}
In this paper a novel algorithm is presented for frequency estimation of a complex exponential by interpolating Fourier coefficients in a non-iterative way by shifting the minimum variance and using a non-linear function. The estimator presented is unbiased and normally distributed with asymptotic variance that is $\pi^{4} / 96 \approx 1.0147$ of the Cramer RaoBound and is optimally uniform over the entire range while requiring calculation of the fewest Fourier coefficients.
\end{abstract}

Keywords-Complex exponential, Frequency estimation, Interpolation of Fourier coefficients and Parameter estimation

\section{INTRODUCTION}

The problem of estimating the frequency of a sinusoidal signal in noise is of prime importance in many applications such as biomedical instrumentation, sonar, communication and defence-related signal processing. This is a fundamental problem and has remained a major topic of research in literature; its importance can be seen in [1] and [2]. The fundamental aspects associated with an optimum frequency estimator are that it should be unbiased, have minimum uniform variance and it should have low computational load. If such an unbiased estimator is possible then the minimum variance of it is given by the Cramer Rao-Bound (CRB), derived by Rife and Boorstyn in [3] for the complex Gaussian independent and identically distributed (i.i.d.) case and is given by:

$$
\sigma_{f}^{2}=\frac{6 f_{s}^{2}}{(2 \pi)^{2} \rho N\left(N^{2}-1\right)}
$$

where $f_{s}$ is the sampling frequency, $\rho$ is the SNR and $N$ is the number of samples of the signal. The frequency domain algorithm that will be presented here is based on the fast Fourier Transform (FFT) and shows good performance at low SNR.

The most common FFT based algorithms employ a two stage approach, using a coarse search followed by a fine search. The coarse search is based on a maximum bin search that is the argument of periodogram maximiser. The fine search can be based on a dichotomous search as in the case of
([4] \& [5]) or interpolation of Fourier coefficients as in the case of ([6] - [9]). The dichotomous search algorithms ([4] \& [5]) are simple but computationally intensive (equivalent to taking an FFT again). These yield an estimator variance that is close to the CRB but are non-uniform over the length of the residual. The estimator based on the interpolation of Fourier coefficients presented in ([6] - [9]) are computationally simple with least computational load, but their variance is still nonuniform. This non-uniform variance can be as high as $\pi^{2} / 6 \approx 1.6449$ when close to the bin center [9]. In [10] and [11], the interpolation algorithms using maximization of non linear function based on Newton method and parabolic fit are presented respectively. The algorithms require zero padding the data to the length $2 N$ and calculation of two additional Fourier coefficients at midpoint frequencies. However, the computational penalty of zero padding the data is significant.

The iterative interpolation algorithm presented by Aboutanios and Mulgrew [12] gives a uniform variance that is $\pi^{4} / 96 \approx 1.0147$ of the CRB. However, it does not use any previously available Fourier coefficients of the FFT for interpolation but calculates four new Fourier coefficients to achieve this performance. In [13] Minhas and Aboutanios resolved this by using more information from FFT that lead to calculation of only two additional Fourier coefficients whilst giving the same performance. Both of these algorithms still do not give a precisely uniform estimation variance of $\pi^{4} / 96 \approx 1.0147$, but deviate from it either close to bin center or at the edge of bin. This deviation in estimator asymptotic variance is more than 1.065 of the $\mathrm{CRB}$. For precisely uniform estimation performance of $\pi^{4} / 96 \approx 1.0147$, both algorithms require further additional calculation of Fourier coefficients that increase the computational load.

In this paper a novel non-iterative algorithm will be presented that does not require zero padding or any intense computational calculation to perform estimation for the residual. Instead it uses already available Fourier coefficients and uses the novel minimum variance shift method, thus resulting in the calculation of the fewest number of Fourier coefficients. In addition to that, the new algorithm also uses a non-linear function to achieve precisely uniform performance of $\pi^{4} / 96 \approx 1.0147$ of the Cramer Rao-Bound. 


\section{FREQUENCY ESTIMATOR}

The algorithm presented here consists of a two stage process: the first is a coarse search based on a periodogram maximiser and the second is a fine search one. This second stage is further divided into two steps: the first utilizes the already available Fourier coefficients and the second requires the additional calculation of Fourier coefficients.

The new algorithm can be seen in table I. The basic interpolation of the Fourier coefficient structure in the proposed algorithm is same as shown in [13]. However, the algorithm presented here integrates a non linear function into the interpolation of Fourier coefficients in the first step of the estimator using three Fourier coefficients. In step two where the Fourier coefficients are again calculated, the estimation is based on interpolation of two Fourier coefficients thus reducing the computational load.

Let us consider the following complex exponential whose frequency has to be estimated.

$$
\begin{aligned}
s(n)=A e^{j\left[2 \pi n \frac{f}{f_{s}}+\theta\right]}+w(n), \\
n=0, \ldots, N-1
\end{aligned}
$$

Here $A$ is the amplitude of the signal, $\theta$ is the phase of the signal and $f$ is the true frequency that has to be estimated. $N$ is the total number of samples available for estimation. The noise term $w(n)$ is zero mean additive white Gaussian with minimum variance of $\sigma^{2}$

The true frequency of the complex exponential signal is given as

$$
f=\frac{\left[m_{N}+\delta_{N}\right]}{N} f_{S}
$$

where $f$ is the true frequency, $m_{N}$ is the true maximum bin and $\delta_{N}$ is the residual within the interval of $[-0.5 \rightarrow 0.5]$. The subscript $N$ shows the dependence of the above on $N$, however for simplification it will be neglected. Stage one comprises the maximum bin search which returns the true maximum bin i.e. $\widehat{m}=m$. The second stage, based on an interpolation of Fourier coefficients, makes an estimate of the residual $\delta$ that is $\hat{\delta}$ in order to determine the true frequency of the signal.

First the interpolation procedure of the Fourier coefficients is discussed. The DFT coefficients of the adjacent bins are given by

$$
Y_{\widehat{m}+a}=\sum_{n=0}^{N-1} s(n) e^{-j 2 \pi n \frac{\widehat{m}+a}{N}} \quad, a= \pm 1
$$

Now substitute the value of a complex signal $s(n)$ in the above equation. For simplicity, suppose that $A=1$ and $f_{s}=1$. The simplification of the above equation yields the following as shown in [13].
TABLE I

NEW FREQUENCY ESTIMATION ALGORITHM

\begin{tabular}{cc}
\hline Let $\quad$ & $\frac{\text { Coarse Search }}{Y=F F T(s)}$ \\
Let $\hat{m}=\operatorname{argmax}_{n}\left\{|Y(n)|^{2}\right\}$
\end{tabular}

Fine Search:

Step 1:

Let

$$
\begin{aligned}
& r_{l}=\operatorname{Re}\left\{Y_{l} Y_{0}^{*}\right\}, \quad l= \pm 1 \\
& \text { if } r_{1}<r_{-1} \\
& \text { then } a=1, \text { else } a=-1
\end{aligned}
$$

$$
\begin{aligned}
\hat{\delta}_{t} & =a \operatorname{Re}\left\{\frac{Y_{\widehat{m}+a}}{Y_{\widehat{m}+a}-Y_{\widehat{m}}}\right\} \\
\hat{\delta}_{c} & =c \operatorname{Re}\left\{\frac{Y_{\widehat{m}+c}}{Y_{\widehat{m}+c}-Y_{\widehat{m}}}\right\} \quad \text { where } c= \pm 1 \\
\hat{\delta}_{o} & =\frac{\hat{\delta}_{+1}+\hat{\delta}_{-1}}{2} \\
& +\left(\hat{\delta}_{+1}-\hat{\delta}_{-1}\right) \frac{3 \hat{\delta}_{t}^{3}+2 \hat{\delta}_{t}}{3 \hat{\delta}_{t}^{4}+6 \hat{\delta}_{t}^{2}+1}
\end{aligned}
$$

Step2: (MVSM)

$$
\begin{aligned}
& \text { if } \hat{\delta}_{o} \geq 0 \\
& \text { then } a=1 \quad \& \quad \beta=-0.5 \\
& \text { else } a=-1 \quad \& \quad \beta=0.5
\end{aligned}
$$

Calculate

$$
\begin{gathered}
Y_{\widehat{m}+l}=\sum_{n=0}^{N-1} s(n) e^{-j 2 \pi n \frac{\widehat{m}+\widehat{\delta}_{o}+\beta+l}{N}}, l=0, a \\
\hat{\delta}=a \operatorname{Re}\left\{\frac{Y_{\widehat{m}+a}}{Y_{\widehat{m}+a}-Y_{\widehat{m}}}\right\}+\hat{\delta}_{o}+\beta
\end{gathered}
$$

Finally

$$
\hat{f}=\frac{\widehat{m}+\hat{\delta}}{N} f_{s}
$$

$$
Y_{\widehat{m}+a}=e^{j \theta} \frac{1-e^{j 2 \pi \delta}}{-j 2 \pi \frac{(\delta-a)}{N}}+W_{a}+O\left(N^{-2}\right)
$$

$$
Y_{\widehat{m}+a}=c \frac{\delta}{(\delta-a)}+W_{a}
$$

In the above approximation the bias is equal to $O\left(N^{-2}\right)$ and the value of $c$ is 


$$
c=-N e^{j \theta} \frac{1-e^{j 2 \pi \delta}}{j 2 \pi \delta}
$$

The fine search uses the following interpolation in the first step of the algorithm (see table I) to calculate the residual $\hat{\delta}$ in the interval of $[-0.5 \rightarrow 0.5]$. First of all a noiseless case is taken where the noise coefficient $W_{a}$ will not be considered.

$$
\hat{\delta}=a \operatorname{Re}\left\{\frac{Y_{\widehat{m}+a}}{Y_{\widehat{m}+a}-Y_{\widehat{m}}}\right\}
$$

Now substitute the value of equation (6) without the noise coefficient in the above equation and solving, which yields

$$
\begin{aligned}
\hat{\delta} & =a \operatorname{Re}\left\{\frac{c \frac{\delta}{(\delta-a)}}{c \frac{\delta}{(\delta-a)}-c}\right\} \\
& =\delta
\end{aligned}
$$

The above estimator $\hat{\delta}$ shows that it can be used to estimate the true residual $\delta$. The decision criterion for selecting the two coefficients being interpolated must be one that has better SNR. One possible criterion is to compare the coefficient on either side of the bin, as used in Rife \& Vincent [7]. This rule gives poor performance as $|\delta| \rightarrow 0$ and is shown in [9]. Thus considering the following rule

$$
\begin{gathered}
r_{l}=\operatorname{Re}\left\{Y_{l} Y_{0}^{*}\right\} \approx \operatorname{Re}\left\{c \frac{\delta}{\delta-l} c^{*}\right\} \\
=|c|^{2} \frac{\delta}{\delta-l}, \quad l= \pm 1
\end{gathered}
$$

From the above, we see that $r_{1} \leq 0 \leq r_{-1}$ for $0 \leq \delta \leq 0.5$ and similarly for $r_{-1} \leq 0 \leq r_{1}$ it is $-0.5 \leq \delta \leq 0$. This justifies the decision rule used in the algorithm.

The detailed theoretical analysis of the interpolation technique of the algorithm based on noise can be seen in [13]. The noise considered in the analysis is independent and identically distributed, with zero mean Gaussian. It has been shown in [13] that the asymptotic distribution of the estimator is similar to the distribution of the noise. Thus the asymptotical performance of the algorithm shows it is unbiased and normally distributed with the variance of the estimator given as

$$
\begin{gathered}
\operatorname{var}[\hat{f}]=\frac{\sigma^{2}(\pi \delta)^{2} f_{s}^{2}}{2 N^{3} \sin ^{2}(\pi \delta)}(1-|\delta|)^{2}\left\{\delta^{2}\right. \\
\left.+(1-|\delta|)^{2}\right\}
\end{gathered}
$$

The best way to check the performance of the minimum variance of asymptotically unbiased estimator is to take its ratio to that of $\mathrm{ACRB}$ given in equation (1) using the value of $\rho=A^{2} / \sigma^{2}$ where $A=1$.

$$
R=\frac{\pi^{4} \delta^{2}}{3 \sin ^{2}(\pi \delta)}(1-|\delta|)^{2}\left\{\delta^{2}+(1-|\delta|)^{2}\right\}
$$

The estimator after step 1 gives a non-uniform variance performance that is minimum as $\pi^{4} / 96 \approx 1.0147$ to ACRB at $|\delta|=0.5$ and maximum of $\pi^{2} / 3 \approx 3.2899$ at $|\delta|=0$. However, the performance of the estimator is worst at the center of the bin $\delta=0$. Quinn in [9] proposed a non-linear function using three Fourier coefficients to improve the above estimator variance in his second algorithm. This non linear function is integrated in step 1 of the algorithm. For this the same interpolation step is repeated to calculate $\hat{\delta}_{+1}$ and $\hat{\delta}_{-1}$ using three Fourier coefficients shown as below

$$
\hat{\delta}_{c}=c \operatorname{Re}\left\{\frac{Y_{\widehat{m}+c}}{Y_{\widehat{m}+c}-Y_{\widehat{m}}}\right\} \text { where } c= \pm 1
$$

The average of $\hat{\delta}_{+1}$ and $\hat{\delta}_{-1}$ gives an estimator whose asymptotic variance is nearly half of $\pi^{2} / 3 \approx 3.2899$ at $\delta=0$ but the asymptotic variance is very high at $\delta= \pm 0.5$. Quinn exploited this minimum variance property at $\delta=0$ and proposed the minimization of the residual error by using $\hat{\delta}=g\left(\hat{\delta}_{+1}, \hat{\delta}_{-1}\right)$ where $g$ is continuous with continuous partial derivative. The value of $g$ is obtained such that it minimizes the asymptotic variance of the estimator. The detailed derivation can be seen in [14] (pp 195-197) and the resulting non-linear function obtained is shown as below

$$
\begin{aligned}
\hat{\delta}_{o}=\frac{\hat{\delta}_{+1}+\hat{\delta}_{-1}}{2} & +\left(\hat{\delta}_{+1}\right. \\
& \left.-\hat{\delta}_{-1}\right) \frac{3 \hat{\delta}_{t}^{3}+2 \hat{\delta}_{t}}{3 \hat{\delta}_{t}^{4}+6 \hat{\delta}_{t}^{2}+1}
\end{aligned}
$$

Where $\hat{\delta}_{t}$ is already calculated by using interpolation shown in equation (7). The ratio of asymptotic variance of using the above non-linear function in the estimator to that of ACRB as shown in [9] is

$$
R=\frac{\pi^{4} \delta^{2}}{6 \sin ^{2}(\pi \delta)} \times \frac{\left(\delta^{2}-1\right)^{2}\left(3 \delta^{4}+1\right)}{3 \delta^{2}+6 \delta^{2}+1}
$$

The minimum asymptotic variance performance achieved after step 1 of the estimator is $(57 / 5504) \pi^{4} \approx 1.0088$ at $\delta= \pm 0.5$ and a maximum variance of $\pi^{2} / 6 \approx 1.6449$ at $\delta=0$. The variance is actually minimized more outside the residual range at $\delta= \pm 0.5217$ that is $57 \pi^{4} / 5516 \approx 1.0066$. The integration of this non-linear function in step 1 improves the estimation at $\delta=0$ and therefore leads to a superior 
performance in step 2 compared to other algorithms that will become evident in simulations.

In the next section is discussed step 2 of the fine search; it will show how the novel technique presented in this paper manipulates the interpolation procedure to give a optimally uniform minimum asymptotic variance for the algorithm that is 1.0147 of the $\mathrm{CRB}$ throughout the estimated residual $[-0.5 \rightarrow 0.5]$.

\section{MINIMUM VARIANCE SHIFT METHOD}

In this section, the minimum variance shift method (MVSM) applied in step 2 of the fine search in the algorithm will be discussed. The MVSM improves the performance of the algorithm by running it again where the estimator has minimum variance. For this, first the residual being calculated in step one is removed, so in order to run the algorithm, $\delta=0$ is taken as a fixed point. In this step algorithm converges to a fixed point following the fixed point theorem ([15], pp.109111). However as stated earlier the algorithm gives the worst estimation in terms of variance at this point. So, taking the point of minimum variance that in our case is at $\delta= \pm 0.5$ and incorporating a parameter $\beta=\mp 0.5$ in the calculation, the Fourier coefficients are as shown below.

$$
Y_{\widehat{m}+l}=\sum_{n=0}^{N-1} s(n) e^{-j 2 \pi n \frac{\widehat{m}+\widehat{\delta}_{o}+\beta+a}{N}}, l=0, a
$$

Prior to the above, the decision criterion is also changed and now if $\hat{\delta}_{o} \geq 0$ then $a=1 \& \beta=-0.5$ else $a=$ $-1 \& \beta=+0.5$. The final residual can be obtained through the following interpolation structure

$$
\hat{\delta}=a \operatorname{Re}\left\{\frac{Y_{\widehat{m}+a}}{Y_{\widehat{m}+a}-Y_{\widehat{m}}}\right\}+\hat{\delta}_{o}+\beta
$$

By doing this, the minimum variance point is shifted to become the fixed point of the algorithm thus resulting in a minimum uniform variance throughout the length of residual. In the above algorithm the minimum variance of the estimator to that of the ACRB is of $\pi^{4} / 96 \approx 1.0147$ at $\delta= \pm 0.5$. This results in an optimally uniform variance of $\pi^{4} / 96 \approx 1.0147$ over the entire residual interval of $[-0.5 \rightarrow 0.5]$. It is important to note that MVSM is not confined to $\delta= \pm 0.5$ but works for minimum variance present anywhere between the residual $\{\delta ;|\delta|=0 \rightarrow 0.5\}$ and it surely converges to that variance. It can also be seen that non-linear function is not used in step 2 since it would require the calculation of third Fourier coefficient and will increase the computational load.

\section{SIMULATION RESULTS}

The new algorithm presented in this paper was simulated. The statistical analysis of the algorithm was undertaken with 10,000 Monte Carlo runs and compared to the theoretical curves obtained in the previous section. The simulation was performed at a sampling frequency of $f_{s}=100 \mathrm{~Hz}$ and the number of samples used was $N=1024$. Fig. 1 shows the ratio of asymptotic estimator variance of the algorithm after step 1 with and without non-linear function (NLF) to that of the Cramer-Rao Bound over the entire range of residual $\delta$. The theoretical curves have also been plotted.

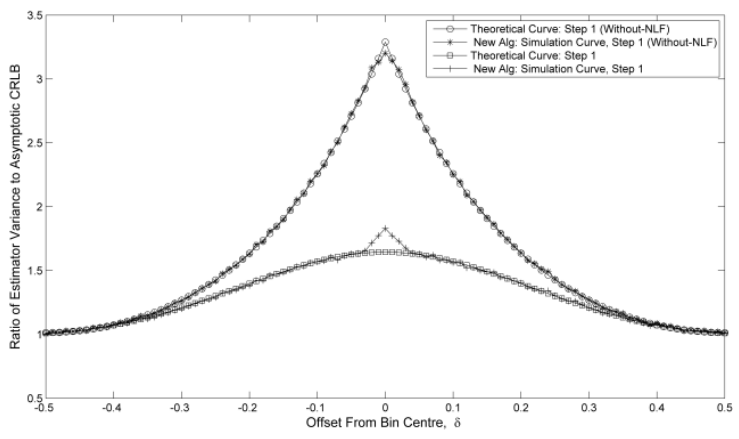

Figure 1. Plot of the ratio of estimator variance of the algorithm with and without non-linear function (NLF) to the asymptotic Cramer Rao-Bound as a function of residual $\boldsymbol{\delta}$. The theoretical curves are also shown. For the simulation, 10,000 Monte Carlo runs were used at an SNR value of 0dB

Fig. 2 shows that the new algorithm after step 2 (MVSM) shows optimal uniform performance of $\pi^{4} / 96 \approx 1.0147$ over the entire residual range. The superior performance of the new algorithm can be seen in figure 2 compared with other algorithms. The Aboutanios and Mulgrew (A\&M) [12] algorithm deviates from the uniform performance at the edge of the bin $|\delta| \rightarrow 0.5$, where as Minhas and Aboutanios (M\&A) [13] algorithm deviates at the center of the bin $|\delta| \rightarrow 0$. This deviation in the asymptotic variance of both the estimators is approximately 1.065 to CRB.

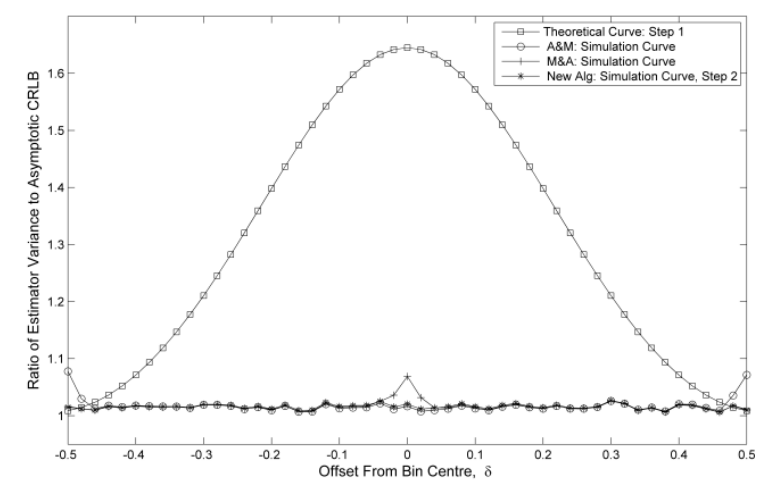

Figure 2. Plot of the ratio of estimator variance of the new algorithm to the asymptotic Cramer Rao-Bound as a function of residual $\boldsymbol{\delta}$. The theoretical curve is shown and the simulation curves of A\&M and M\&A algorithms are also shown. For the simulation, 10,000 Monte Carlo runs were used at an SNR value of $0 \mathrm{~dB}$

Figure 3 shows the estimation errors in $\mathrm{Hz}$ of the new algorithm in terms of standard deviation over an SNR range of $[-20 d B \rightarrow 0 d B]$. The residual $\delta$ selected was random but away from the center and the edge of the bin (discussed earlier). The new algorithm presented here shows similar performance following the CRB curve and is identical to the A\&M and M\&A algorithms. However the performance of $A \& M$ is worst at the edge of the bin and M\&A is at the center of the bin. The superior performance of the new algorithm can 
be seen in figure 4 where residual selected is $|\delta|=0.5$ and $\delta=0$.

It is quite evident from the figures that the asymptotic variance of the new algorithm is marginally above the Cramer Rao-Bound.

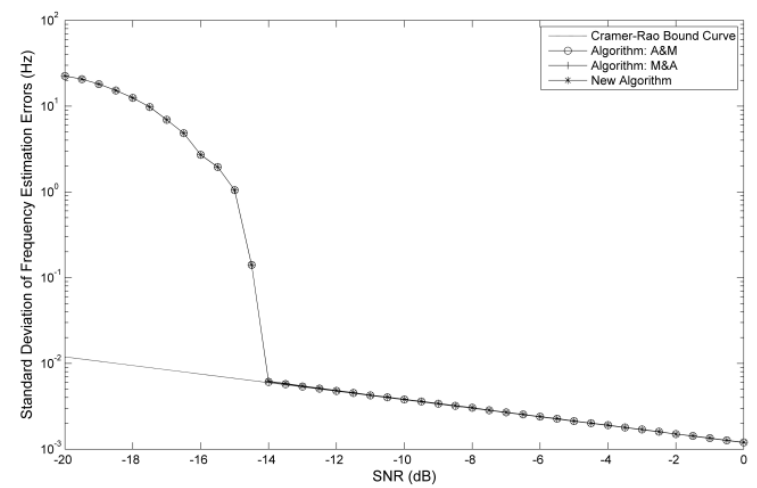

Figure 3. Plot of standard deviation of frequency estimation errors of the new algorithm as a function of SNR range of $[-20 d B \rightarrow 0 d B]$. The residual used is random but away from the center and the edge of the bin. A comparison is also shown with the A\&M and M\&A algorithms. The Cramer Rao-Bound is also shown. For simulation 10,000 Monte Carlo runs were used.

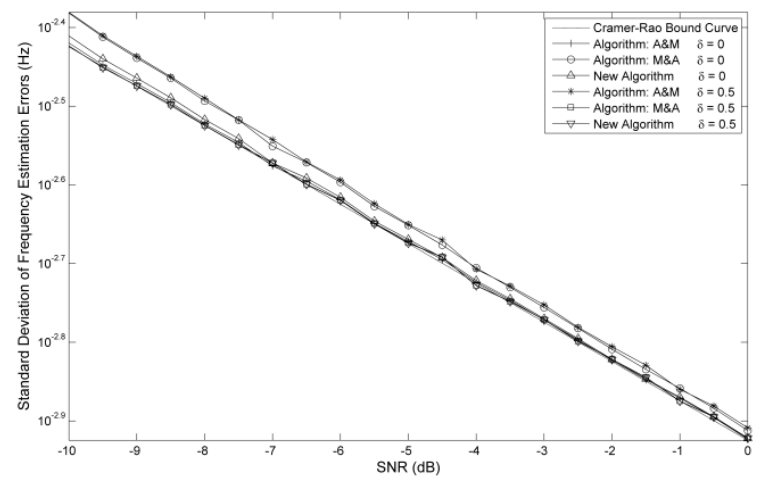

Figure 4. Plot of standard deviation of frequency estimation errors of the new algorithm as a function of SNR range of $[-\mathbf{1 0 d B} \rightarrow \mathbf{0 d B}]$. A comparison is also shown with the A\&M and M\&A algorithms. The Cramer Rao-Bound is also shown. For simulation 10,000 Monte Carlo runs were used.

\section{DISCUSSION}

In this section a computational comparison with other algorithms will be discussed. The algorithm presented here requires the calculation of fewer DFT (discrete Fourier Transform) coefficients, i.e only two to give a uniform estimator performance of $\pi^{4} / 96 \approx 1.0147$ to that of ACRB. The computational load of the algorithm is not the interpolation but the calculation of DFT coefficients that is done separately to the FFT of the signal. So, the computational load of the algorithm apart from the FFT is $(4 N-2)$ operations. Each DFT coefficient requires $N$ multiplications and $N-1$ additions. It is shown in [12] that Aboutanios \& Mulgrew algorithm converges after two iterations, thus requiring a calculation of four DFT coefficients. This yields a computational load of $(8 N-4)$ operations. The Minhas and Aboutanios algorithm [13] requires the calculation of only two DFT coefficients as in the new algorithm, but deviates from the uniform variance performance of the estimator (discussed earlier).

The estimator presented in this paper not only requires the smallest computational load of $(4 N-2)$ operations in fine search, but will also give an optimally uniform performance that is $\pi^{4} / 96 \approx 1.0147$ of the CRB. This is superior to the performance shown by other algorithms. However, both the Aboutanios and Mulgrew and also the Minhas and Aboutanios algorithms are iterative in nature. So by increasing one more iteration can achieve the same performance as that of the new algorithm but at an increased computational load. For Aboutanios and Mulgrew, it would require three iterations with calculation of six DFT coefficients and would result in a computational load of $(12 N-6)$ operations. Similarly for Minhas and Aboutanios algorithm it would be $(8 N-4)$ operations.

Computational efficiency is of primary importance in the consideration of real-time performance. In many applications of DSP, it is not feasible to conduct off-line processing, for example with respect to voice communications, live performance and noise cancellation. Optimization of the algorithm with respect to efficiency ensures that, for a given processor, appropriate frequency estimation can be achieved within the time constraints of the system.

\section{CONCLUSION}

In this paper a novel algorithm that gives an optimally uniform frequency estimation performance over the entire residual range is presented. It has been shown theoretically through analysis and practically through simulations that the asymptotic variance of the estimator of the algorithm to that of $\mathrm{ACRB}$ is $\pi^{4} / 96 \approx 1.0147$. The algorithm requires the calculation of the fewest Fourier coefficients, i.e. only two. The algorithm presented here gives superior performance compared to other interpolation algorithms with the smallest computational load.

\section{REFERENCES}

[1] B. Boashash, "Estimating and interpreting the instantaneous frequency of a signal II. algorithms and applications", Proceeding of the IEEE, vol. 80 , no. 4 , pp. 540-568, 1992.

[2] M. A. Richards, "Fundamentals of Radar Signal processing", New York: McGraw-Hill, 2005.

[3] D. C. Rife and R. R. Boorstyn, "Single tone parameter estimation from discrete time observations", IEEE Transaction on Information Theory, vol. IT-20, no. 5, pp. 591-8, 1974.

[4] Y. V. Zakharov and T. C. Tozer, "Frequency estimator with dichotomous search of periodogram peak", IEEE Electronics letter, vol. 35 , no 19 , pp. 1608-1609, 1999.

[5] E. Aboutanios, "A modified dichotomous search frequency estimator", IEEE Signal Processing Letters, vol. 11, no. 2, pp. 186-188, 2004.

[6] Barry G. Quinn, "Estimating frequency by interpolation using Fourier coefficients", IEEE Transaction on Signal Processing, vol. 42, no. 5, pp. 1264-1268, 1994.

[7] D. C. Rife and G. A. Vincent, "Use of the discrete Fourier transform in the measurement of frequencies and levels of tone", Bell Syst. Tech. J, vol. 49, no.2, pp. 197-228, Feb 1970. 
[8] M. D. Macleod, "Fast nearly ML estimation of the parameter of real or complex single tones or resolved multiple tones", IEEE Transactions on Signal Processing, vol. 46, no. 1, pp. 141-8, 1998.

[9] Barry G. Quinn, "Estimation of frequency, amplitude and phase from the DFT of a time series", IEEE Transactions on Signal Processing, 45, 814817, 1997.

[10] Barry G. Quinn, "Recent advances in rapid frequency estimation", Elsevier Digital Signal Processing, vol. 19, no. 6, pp. 942-948, 2009.

[11] E. Aboutanios, "Generalised DFT-Based Estimators of the frequency of a complex exponential in noise", IEEE International Congress on Image and Signal Processing (CISP 2010), pp. 2998-3002, 2010.
[12] E. Aboutanios and B. Mulgrew, "Iterative frequency estimation by interpolating on Fourier coefficients", IEEE Transactions on Signal Processing, vol. 53, no. 4, pp. 1237-1242. April 2005.

[13] S. Minhas and E. Aboutanios, "Estimation of the frequency of a complex exponential", IEEE International Conference on Acoustic, Speech and Signal Processing (ICASSP 2008), pp. 3693-3696, April 2008.

[14] Barry G. Quinn and E. J. Hannan, "The estimation and tracking of frequency", Cambridge University Press, New York, 2001.

[15] R. Plato, "Concise numerical mathematics", American Mathematical Society, 2003. 